\title{
ÉTUDE DE QUELQUES RELATIONS ENTRE LE MODE DE CONSERVATION DU FOURRAGE INGÉRÉ ET LE COMPORTEMENT ALIMENTAIRE ET MÉRYGIQUE DES MOUTONS
}

\author{
J.-P. DULPHY \\ avec la collaboration technique de J.-M. Boisseav, H. Bousguet et L. L'Hotelier \\ Station de Recherches sur l'Élevage des Ruminants, \\ Centre de Recherches de Clermont-Ferrand, I. N.R. A., \\ 63 - Saint-Genès-Champanelle
}

\section{RÉSUMÉ}

I. Nous avons étudié l'influence du mode de conservation des fourrages sur les manifestations du comportement alimentaire et mérycique de moutons au cours de deux cssais.

- Dans le premier essai, 5 moutons ont reçu un foin et un ensilage de fétuque élevée de digestibilités proches au cours de 6 périodes successives selon trois méthodes : changements brusques de régime entre le foin et l'ensilage distribués ad libitum; distribution en quantités limitées à un même niveau d'ingestion du foin et de l'ensilage; distribution le même jour du foin et de l'ensilage, soit séparément, soit en même temps.

- Dans le deuxième essai, 6 moutons ont reçu une même plante, la fléole, d'une part sous forme de fourrage vert, d'autre part sous forme de trois fourrages conscrvés, un foin et deux ensilages. Les comparaisons ont été faites pour des distributions à volonté, puis en quantité limitée.

2. La durée journalière d'ingestion est plus faible pour les ensilages que pour le fourrage vert ou pour le foin préparés à partir de la même plante sur pied (tabl. 2 et 6). Les repas d'ensilage sont plus courts et plus nombreux que ceux de foin ou de fourrage vert.

3. La vitesse d'ingestion (inverse de la durée unitaire d'ingestion) des ensilages est variable : l'ensilage est ingéré moins rapidement que le foin dans le premier essai et à la même vitesse dans le second essai.

4. Lorsque les animaux ont reçu sans transition de l'ensilage après un régime de foin, les quantités de matière sèche ingérées ont diminué progressivement durant 4 jours tandis que la durée unitaire d'ingestion et le nombre de repas augmentaient orutalenent (fig. I). Lorsque les animaux ont reçu de nouveau du foin les quantités de matière sèche ingérées ont réaugmenté immédiatement ainsi que la durée des repas.

5. Lorsque les fourrages sont ingérés à volonté la durée de rumination est relativement constante; elle ne semble pas être un facteur limitant de la quantité d'ensilage ingérée. Des quantités faibles et égales de fourrages conservés différcmment sont ruminées de la même manière. La durée des cycles de rumination est plus faible avec l'ensilage ou avec le fourrage vert qu'avec le foin. 


\section{INTRODUCTION}

De nombreuses études entreprises aux U. S. A. (MoOre et al., I960; THomas ct al., I960; WALDO et al., I966), en Grande-Bretagne (HARRIS et al., I966 ; MCLEOD et al., I970), en Irlande (MCCARRICK et al., I965) et en France (DEMARquiliy et Weiss, I970; Demarquilly et JARRIGE, I970) ont montré que les ensilages de graminées ou de légumineuses sont ingérés en quantité nettement plus faible que les fourrages verts ou les foins correspondants. La diminution de quantité ingérée entre la plante sur pied et la plante ensilée s'observe même pour les ensilages très bien conservés dont la digestibilité est identique à celle du fourrage vert initial.

Les causes de cette diminution sont mal connues et vraisemblablement complexes. Moore, Thomas et Sykes (Ig60) pensent que le goût et l'odetır de l'ensilage n'auraient pas d'action importante mais que l'ensilage agirait directement sur l'appétit des animaux. Ces auteurs suggèrent une action de corps azotés provenant de la dégradation des matières azotées de la plante ensilée et estiment que les acides de l'ensilage n'agissent que pour une légère part. MCCARRICK et al. (I965) pensent plutôt qu'un déséquilibre acido-basique introduit par la consommation d'ensilage serait à l'origine des faibles quantités ingérées. Plus récemment MC LEOD et al. (I970) concluent, à l'inverse des études américaines, que les acides produits durant la fermentation normale de l'ensilage peuvent limiter les quantités ingérées.

Afin de savoir comment les animaux réagissent face à l'apport d'ensilage et de rapprocher éventuellement ces observations des hypothèses précédentes, nous avons enregistré les manifestations du comportement alimentaire et mérycique des moutons selon la technique de Ruckebusch (I953). Lin effet, il existe des liaisons positives entre les durées d'ingestion et de rumination et les quantités de fourrage ingérées par les animaux (GoRion, I965; WELCH et SMITH, I969 a et $b$; KERBAA, 1969). Le comportement des animaux a été examiné au cours de deux essais. Dans le premier, un ensilage a été comparé à un foin de même origine : les deux fourrages ont été distribués selon certaines modalités propres à observer un comportement différent des animaux. Dans le second, nous avons cherché à savoir dans quelle mesure 1'ingestion et la rumination étaient influencées par le mode de conservation du fourrage en essayant de connaître la part qui revient respectivement au mode de conservation proprement dit et aux variations du niveau d'ingestion.

\section{MATÉRIEL ET MÉTHODES}

\section{Premier essai}

Nous avons choisi parmi les fourrages conservés dont la digestibilité et la quantité ingérée avaient été mesurées sur des moutons durant l'hiver 1969-1970, un foin et un ensilage de fétuque élevée de digestibilité très voisine, provenant de la même plante fauchée au stade épiaison. Le foin avait d'abord été séché au sol, puis ventilé en grange. L'ensilage avait été récolté avec une machine à couteaux et conservé sans préfanage, après addition d'acide formique ( $\mathbf{I}, 5$ litre/tonne cle matière fraîche) ; sa conservation était satisfaisante. $L_{\exists}$ digestibilité de la matière sèche de 
cet ensilage était plus faible que celle du foin d'environ 2 points $(61,0$ contre 62,6$)$, mais elle était légèrement sous-estimée n'ayant pas été corrigée pour les pertes de matières volatiles à l'étuve. Les caractéristiques de ces deux fourrages sont présentées dans le tableau $\mathbf{I}$.

Les animaux ayant servi à cette étude sont 5 moutons mâles castrés de race Texel, d'un poids moyen égal à $53 \mathrm{~kg}$, qui n'avaient jamais reçu d'ensilage avant l'expérience.

Trois méthodes ont été employées successivement pour comparer les réactions des animaux vis-à-vis du foin et de l'ensilage :

$I^{0}$ changements brusques de régime entre le foin et l'ensilage. Les animaux ont d'abord reçu à volonté (Io à $15 \mathrm{p}$. Ioo de refus) en 2 repas par jour, à $8 \mathrm{~h}$ et à $\mathrm{I} 7 \mathrm{~h}$, le foin (jours I à I6 - période I), puis l'ensilage (jours I 7 à 37 - période 2), puis de nouveau le foin (jours 38 à 47 - période 3);

$2^{\circ}$ distribution en quantités limitées à un même niveau d'ingestion du foin, puis de l'ensilage. Les animaux ont reçu, toujours en 2 repas, une quantité limitée à $35 \mathrm{~g}$ de matière sèche par $\mathrm{kg}$ $\mathrm{P}^{0,75}$ et par jour de foin (jours 48 à 61 - période 4 ), puis d'ensilage (jours 62 à 75 - période 5 ) ;

$3^{\circ}$ distribution le même jour du foin et de l'ensilage. Durant la période 6 (jours 76 à 82), les animaux ont reçu, le matin $20 \mathrm{~g}$ de matière sèche de foin par $\mathrm{kg} \mathrm{P}^{\mathbf{0}, 75}$, puis une quantité non limitée d'ensilage pour le reste de la journée. Enfin, dans une dernière période (jours 83 à 86 ), le foin et l'ensilage ont été distribués simultanément à volonté pendant 4 jours.

\section{Second essai}

A partir d'un même fourrage vert de fléole récolté au stade épiaison du premier cycle d'exploitation, un foin et deux ensilages ont été préparés. Le foin a d'abord été préfané sur le champ, puis séché par ventilation en grange. Les đeux ensilages, récoltés sans préfanage avec une machine à couteaux, ont été conservés en silos hermétiques, l'un avec 2 litres d'acide formique par tonne et l'autre sans acide. La composition chimique de ces fourrages est rapportée au tableau I, ainsi que la digestibilité de leur matière organique mesurée avec des moutons.

Ces derniers sont 6 moutons mâles castrés de race Texel pesant de 50 à $60 \mathrm{~kg}$. Ils ont reçu le fourrage vert récolté chaque jour durant le mois de juin r97o et les trois fourrages conservés durant l'hiver suivant. Dans chaque cas les animaux ont reçu le fourrage à volonté pendant Io jours, puis ont été mis à la diète pendant 48 heures. Après cette période de jeûne, trois moutons ont reçu un repas unique représentant $25 \mathrm{p}$. Ioo de la quantité journalière de matière sèche qu'ils avaient ingérée sous forme de fourrage vert et les trois autres moutons 50 p. Ioo.

TABIEAU I

Caractéristiques des fourrages utilisés

\begin{tabular}{|c|c|c|c|c|c|c|}
\hline & \multicolumn{2}{|c|}{ Essai 1} & \multicolumn{4}{|c|}{ Essai 2} \\
\hline & Ensilage & Foin & $\begin{array}{c}\text { Fourrage } \\
\text { vert }\end{array}$ & Foin & $\begin{array}{l}\text { Ensilage } \\
+ \text { acide }\end{array}$ & $\begin{array}{l}\text { Ensilage sans } \\
\text { conservateur }\end{array}$ \\
\hline $\begin{array}{l}\text { Digestibilité de la matière } \\
\text { organique } \ldots \ldots \ldots \ldots \ldots \\
\text { Teneur en matière sèche } \ldots\end{array}$ & $\begin{array}{l}60,9 \\
22,0\end{array}$ & $\begin{array}{l}63,0 \\
86,0\end{array}$ & $\begin{array}{l}63,9 \\
17,0\end{array}$ & $\begin{array}{l}61,2 \\
89,6\end{array}$ & $\begin{array}{l}60,7 \\
16,3\end{array}$ & $\begin{array}{l}60,6 \\
15,7\end{array}$ \\
\hline $\begin{array}{l}\text { Composition chimique (en } \\
\text { p. } 100 \text { de la matière sèche) } \\
\text { Matières azotées } \ldots \ldots \ldots \\
\text { Cellulose brute } \ldots \ldots \ldots \ldots \\
\mathrm{NH}_{3} \ldots \ldots \ldots \ldots \\
\text { Acide lactique } \ldots \ldots \ldots \ldots \\
\text { Acide acétique } \ldots \ldots \ldots \ldots \\
\text { Acide butyrique } \ldots \ldots \ldots\end{array}$ & $\begin{array}{c}9,9 \\
33,6 \\
0,23 \\
5,56 \\
1,29 \\
0,00\end{array}$ & $\begin{array}{l}12,3 \\
29,7 \\
- \\
- \\
-\end{array}$ & $\begin{array}{c}10,4 \\
35,6 \\
- \\
- \\
-\end{array}$ & $\begin{array}{r}9,0 \\
36,9 \\
- \\
- \\
- \\
-\end{array}$ & $\begin{array}{r}8,2 \\
41,3 \\
0,10 \\
3,55 \\
1,53 \\
0,12\end{array}$ & $\begin{array}{r}7,6 \\
41,2 \\
0,41 \\
1,19 \\
4,28 \\
1,05\end{array}$ \\
\hline
\end{tabular}


MESURES

Les quantités journalières de matic̀re sèche ingérées par chaque mouton ont été mesurées par pesée des quantités distribuées et refusées et détermination par séchage, dans une étuve ventilée réglée à $80^{\circ} \mathrm{C}$, des tencurs en matière sèche des fourrages distribués à chaque repas.

Dans le premier essai, les manifestations du comportement alimentaire et mérycique des animaux ont été enregistrées clurant plusieurs jours consécutifs, après chaque changement de régime, puis au cours de chaque période, lorsque les quantités ingérées par les animaux s'étaient stabilisées (cf. fig. r). Dans le sccond essai, les enregistrements ont été effectués pendant 3 jours avant la mise à la diète pour pouvoir comparer lo comportement des animaux nourris à volonté, puis pendant les 2 jours de mise à la diète, et enfin pendant 2 jours à la suite de la distribution du repas expérimental unique. Nous appellerons cluréc unitaire d'ingestion ou de rumination le temps passé à ingérer ou à ruminer par $\mathrm{g}$ consommé (par $\mathrm{kg}$ de poids métaboliquc); la durée de mastication est la somme des clurées d'ingestiqn et cle rumination (cf. DuLPHy, I971).

Le temps de séjour moyen clans le rumen et dans le tube digestif de la fraction indigestible du foin ou de l'ensilage (essai I) a été mesuré sur 2 des 5 moutons, selon la technique des particules colorées de BaLCH (1950) pendant les périodes 2, 3 et 4 du premicr essai.

\section{RÉSUL,TATS}

\section{Essai 1}

Changements brusques de régime.

Quand les moutons sont passés brutalement du foin à l'ensilage, tous deux distribués à volonté, la quantité de matière sèche ingérée a diminué progressivement pour se stabiliser au bout de 5 jours à un niveau inférieur de 24,6 p. Ioo à celui enregistré pour le foin (fig. I). La durée journalière d'ingestion, la durée unitaire d'ingestion et le nombre de repas ont brutalement augmenté le premier jour, puis ont diminué durant les jours suivants. La durée de rumination, le nombre de périodes de rumination par 24 heures n'ont guère été modifiés mais la durée des cycles de rumination a diminué et la durée unitaire de rumination a augmenté (tabl. 2, fig. I).

Lorsque le niveau d'ingestion est stabilisé, les moutons font un plus grand nombre de repas lorsqu'ils reçoivent l'ensilage que lorsqu'ils reçoivent le foin, mais, les repas étant beaucoup plus courts, ils passent moins de temps à ingérer par 24 heures et avec une durée unitaire d'ingestion plus grande. La durée journalière et le nombre des périodes de rumination sont peu modifiés, alors que la durée unitaire de rumination est beaucoup plus élevée.

Quand les animaux ont à nouveau reçu du foin sans transition, la quantité ingérée a augmenté brutalement et s'est stabilisée très vite. Les différents paramètres du comportement ont retrouvé très rapidement des valeurs voisines de celles qu'ils avaient lors de la première période de distribution du foin. Cependant, le nombre de repas a été plus élevé que durant la première période et n’a diminué que très lentement; de même, il existe une légère différence quant à la durée journalière d'ingestion. Cela s'explique vraisemblablement par le fait que les animaux ont ingéré 
plus de matière sèche que durant la première période $\left(5 \mathrm{I}, 3\right.$ contre $\left.45,8 \mathrm{~g} / \mathrm{kg} \mathrm{P}^{0,75}\right)$ pour compenser une sous-alimentation lors de la période de distribution de l'ensilage. Par contre, la durée des repas principaux a retrouvé très vite une valeur voisine de celle de la première période.

TABLEAU 2

Essai 1: comportement alimentaire des moutons

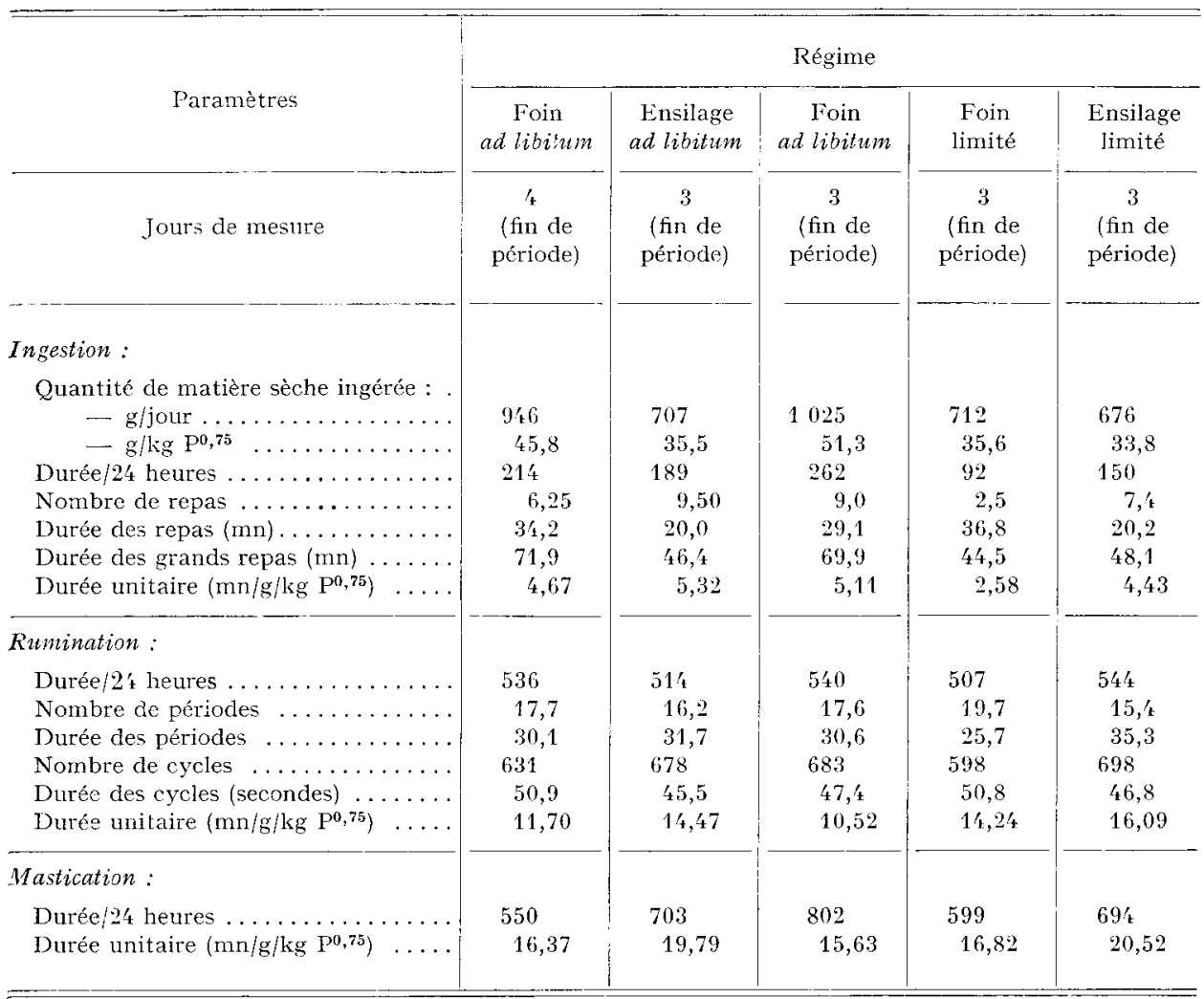

Le comportement des animaux après la distribution du repas du matin entre $8 \mathrm{~h}$ et II h a été examiné plus en détail. C'est ainsi que 36 à $39 \mathrm{p}$. Ioo de la quantité journalière d'ensilage ingérée sont consommés pendant ces 3 heures, avec une durée unitaire équivalente à celle calculée pour la journée. Ces valeurs sont les mêmes pour le foin à partir du troisième jour (tabl. 3).

Comparaison lorsque le foin et l'ensilage sont ingérés en quantité limitée et égale.

L'ingestion de foin a été très peu fractionnée (repas peu nombreux et longs) et rapide (durée unitaire d'ingestion plus faible) par rapport à celle de l'ensilage. Ia durée unitaire de rumination a été légèrement plus faible pour le foin que pour 1'ensilage, tandis que les cycles de rumination étaient plus longs (tabl. 2). 
TABLEAU 3

IEssai 1 : comportement lors du repas principal (passage ensilage-foin)

\begin{tabular}{|c|c|c|c|c|c|c|c|c|c|}
\hline Ration & \multicolumn{3}{|c|}{ Ensilage } & \multicolumn{6}{|c|}{ Foin } \\
\hline Jour & 35 & 36 & 37 & 38 & 39 & 40 & 41 & 42 & 43 \\
\hline Quantité ingérée le matin $(\mathrm{g})$. & 291 & 264 & $2 / 40$ & 498 & 412 & 386 & 387 & 417 & 366 \\
\hline P. 100 total journalier & 39 & 37 & 36 & 45,4 & 40 & 38,5 & 37,3 & 41,0 & 36,3 \\
\hline Quantité ingérée journalière $(g)$ & 740 & 708 & 670 & 1096 & 1023 & 1010 & 1037 & 1019 & 1015 \\
\hline $\begin{array}{l}\text { Durée unitaire d'ingestion } \\
\left(\mathrm{mn} / \mathrm{g} / \mathrm{kg} \mathrm{P}^{0,75}\right):\end{array}$ & & & & & & & & & \\
\hline entre $8 \mathrm{~h}$ et $11 \mathrm{~h}$ & 5,22 & 4,92 & 6,70 & 4,48 & 4,10 & 4,50 & 6,00 & 5,32 & 5,16 \\
\hline sur $24 \mathrm{~h} \quad \ldots \ldots$ & 5,52 & 5,46 & 6,32 & 5,16 & 5,10 & 5,10 & 5,20 & 5,38 & 5,16 \\
\hline
\end{tabular}

Association foin-ensilage.

Le foin distribué en quantité limitée est ingéré rapidement, pratiquement en un seul repas, tandis que l'ingestion d'ensilage demeure lente et fractionnée. Finalement, les quantités totales de matière sèche ingérées sont égales à celles ingérées durant la première période de foin (tabl. 4). Si on donne le libre choix aux animaux

TABLEAU 4

Essai 1 : association foin-ensilage

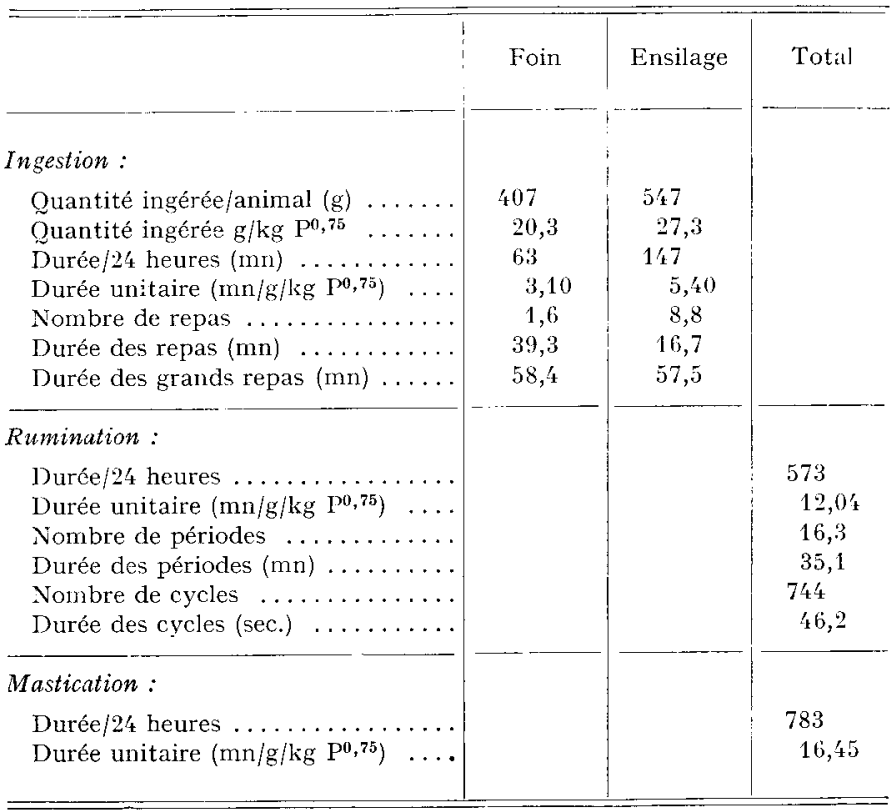


entre le foin et l'ensilage, l'ingestion d'ensilage diminue rapidement et l'ingestion totale de matière sèche augmente (fig. I).

\section{Temps de séjour de l'indigestible dans le rumen et le tube digestif.}

Pour des quantités ingérées très comparables le temps de séjour de l'ensilage est sensiblement plus court que celui du foin, mais la différence est faible (tabl. 5).

\section{TABIEAU 5}

Tomps de séjour digestif du foin et de l'ensilage mesuré sur 2 moutons

\begin{tabular}{|c|c|c|c|}
\hline & $\begin{array}{l}\text { Période ensilage } \\
\text { ad libitu's }\end{array}$ & $\begin{array}{l}\text { Périods foin } \\
\text { limité }\end{array}$ & $\begin{array}{c}\text { 'Période ensilage } \\
\text { limité }\end{array}$ \\
\hline --.---_---_--- ___ & - & - & \\
\hline $\mathrm{T} ; \mathrm{p} .100$ & $\because h \quad 30$ & $\because 6$ h & :30) h 15 \\
\hline $\mathrm{T} \times 01 \mathrm{p} .100$ & $85 \mathrm{~h} 15$ & $9+h$ h & $88 \mathrm{~h} 30$ \\
\hline $\mathrm{T} \mathrm{N}^{2}-\mathrm{T} 5 \ldots \ldots \ldots$ & $60 \mathrm{~h} \cdot 5$ & $6 x h 45$ & so h 15 \\
\hline$T$ moyeri $\ldots \ldots \ldots \ldots$ & $61 \mathrm{~h} 30$ & $1.7 \mathrm{~h} 15$ & 6,4 h 45 \\
\hline $\begin{array}{l}\text { Quantité de matière sè- } \\
\text { che ingérée pendant la } \\
\text { période de mesure (s) }\end{array}$ & 705 & 711 & 651 \\
\hline
\end{tabular}

\section{Essai2}

I. Fourrages distribués à volonté.

L'ensilage conservé avec de l'acide formique et le foin ont été ingérés en quantité comparable au fourrage vert (tabl. 6). L'ensilage sans conservateur a été, par contre, consommé en quantité significativement plus faible (- I5,6 p. Ioo).

Les durées journalières d'ingestion du foin et des ensilages ont été plus faibles que celle du fourrage vert, ce qui s'est traduit par une durée unitaire d'ingestion plus faible pour les fourrages conservés. Les repas ont été plus courts et plus nombreux pour les ensilages, notamment pour celui ingéré en plus faible quantité ; la durée des repas a été en moyenne de $22,3 \mathrm{mn}$ pour cet ensilage contre $48,3 \mathrm{mn}$ pour le fourrage vert. Les repas les plus longs ont été observés pour le foin (56,8 mn). Pour les trois fourrages conservés la durée de rumination a été plus longue que pour le fourrage vert; la durée unitaire de rumination, plus élevée pour les ensilages que pour le fourrage vert ou le foin, a été maximum pour l'ensilage sans conservateur.

La durée unitaire de mastication a été la même pour le fourrage vert et le foin. Par contre, elle a été beaucoup plus élevée pour les ensilages.

\section{Fourrages distribués en quantité limitée lors d'un repas après la mise à la diète.}

Les durées d'ingestion des quatre fourrages sont reliées très étroitement aux quantités ingérées (tabl. 7). Une analyse de variance montre de plus que les droites de régression liant la durée d'ingestion et la quantité ingérée sont pratiquement 


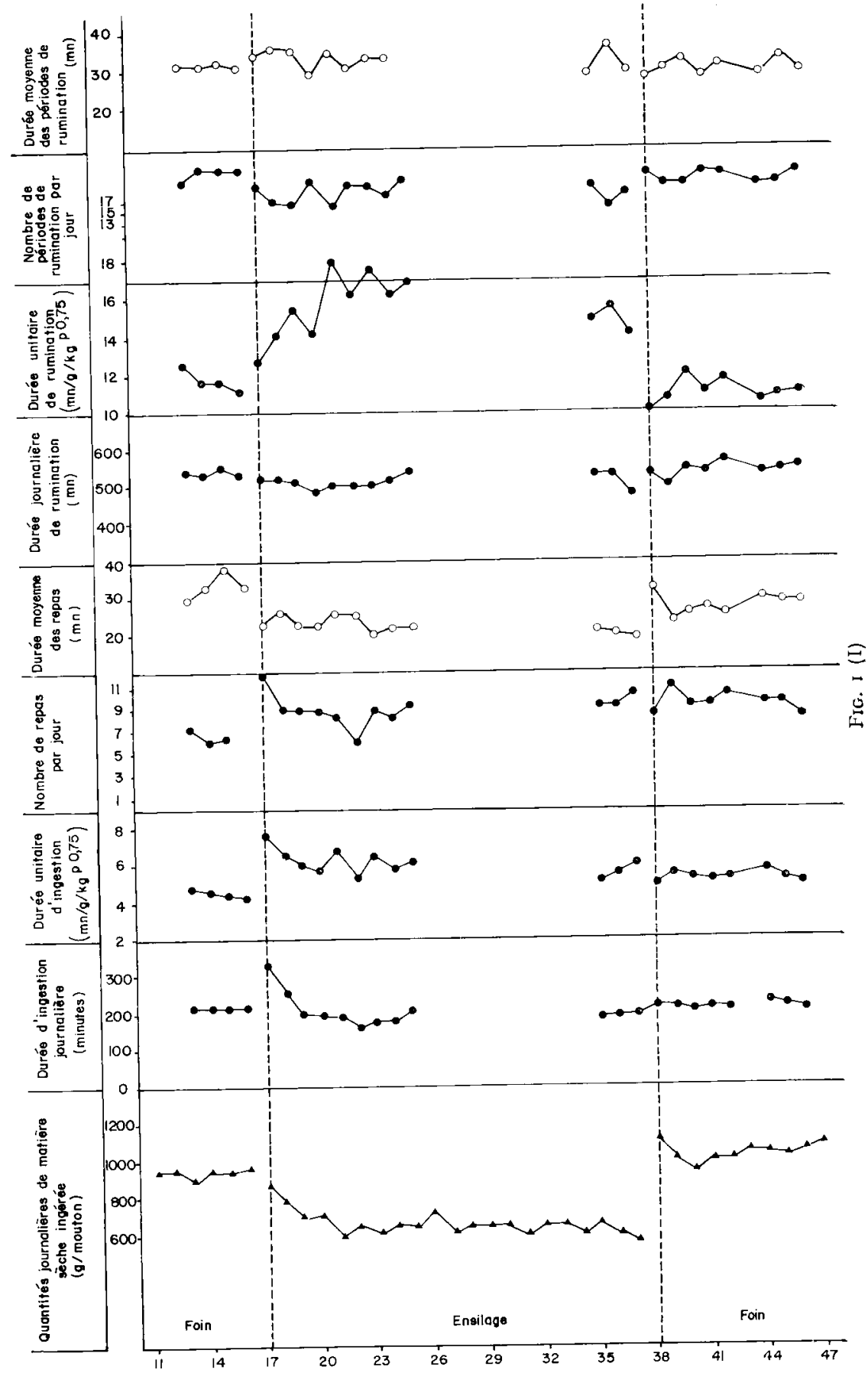




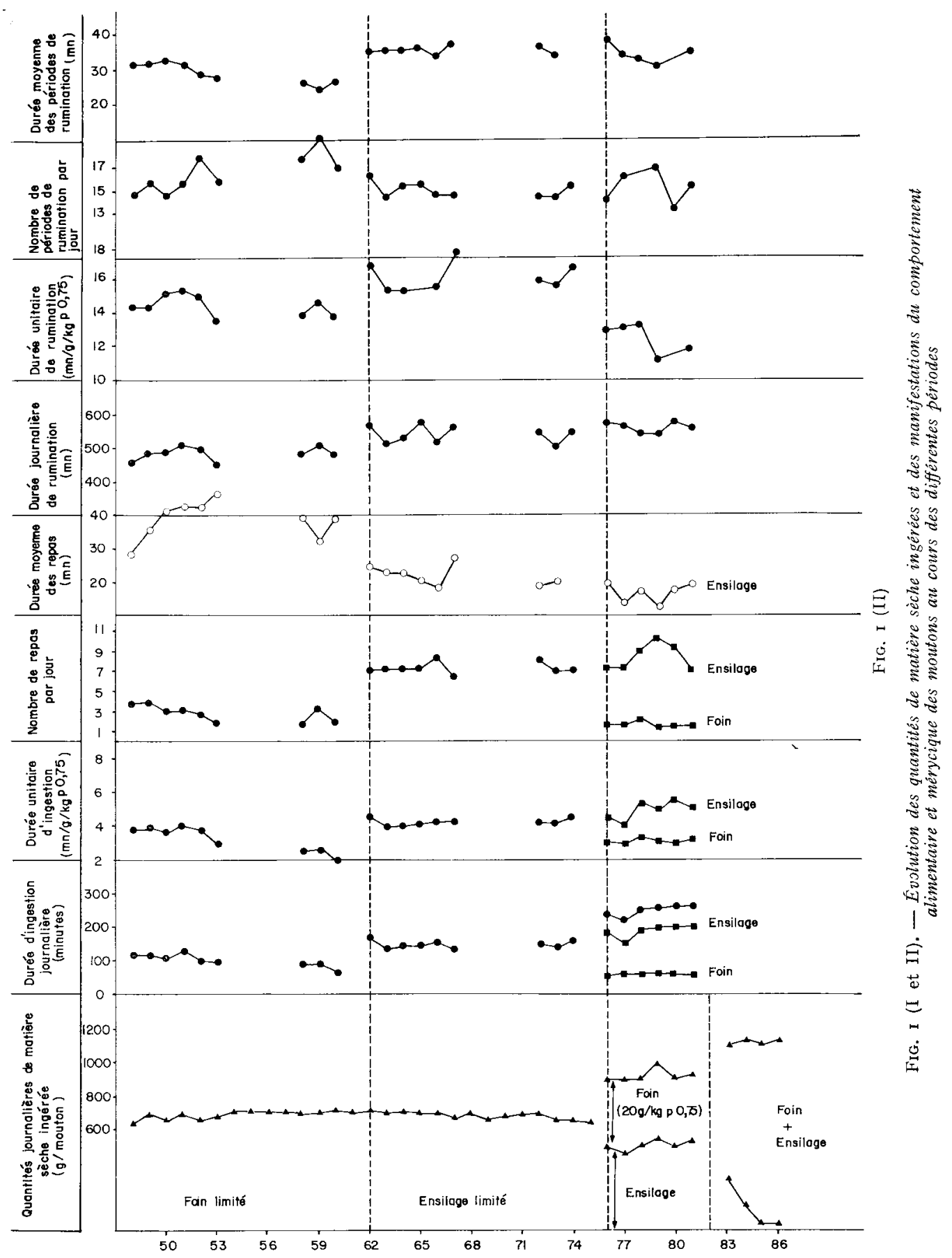




\section{TABLEAU 6}

Essai 2: comparaison des 4 fourrages pour des quantités ingérées ad libitum

Quantités de matière sèche ingérées $\left(g / \mathrm{kg} \mathrm{P}^{0,75}\right) \ldots \ldots \ldots \ldots \ldots \ldots \ldots$

Durée d'ingestion/jour (mn) .......

Durée unitaire d'ingestion $(\mathrm{mn} / \mathrm{g} /$ $\left.\mathrm{kg} \mathrm{P}^{\mathbf{0}, 75}\right) \ldots \ldots \ldots \ldots \ldots \ldots \ldots \ldots \ldots \ldots \ldots \ldots \ldots \ldots \ldots \ldots$

Nombre de repas/jour $\ldots \ldots \ldots \ldots \ldots$

Durée des repas (mn) ...........

Durée des repas suivant une distribution (mn) ..............

Durée de rumination/jour $(\mathrm{mn}) \ldots \ldots$

Durée unitaire de rumination $\left(\mathrm{mn} / \mathrm{g} / \mathrm{kg} \mathrm{\textrm {P } ^ { 0 , 7 5 } )} \ldots \ldots \ldots \ldots \ldots\right.$

Nombre des périodes de rumination .

Durée des périodes de rumination (mn)

Durée des cycles de rumination (sec) .

Durée de mastication/jour $(\mathrm{mn})$....

Durée unitaire de mastication $\left(\mathrm{mng} / \mathrm{kg} \mathrm{P}^{\mathbf{0}, 75}\right)$

\begin{tabular}{|c|c|c|c|}
\hline Fourrage vert & Foin & $\begin{array}{c}\text { Ensilage } \\
+ \\
\text { acide }\end{array}$ & $\begin{array}{c}\text { Ensilage } \\
\text { sans } \\
\text { conservateur }\end{array}$ \\
\hline $\begin{array}{l}46,1 a b \\
287 a\end{array}$ & $\begin{array}{l}48,7 a \\
262 b\end{array}$ & $\begin{array}{l}44,5 b \\
246 b\end{array}$ & $\begin{array}{l}38,9 c \\
212 c\end{array}$ \\
\hline $\begin{array}{l}6,24 a \\
5,8 a b \\
48,3\end{array}$ & $\begin{array}{l}5,51 b \\
4,6 a \\
56,8\end{array}$ & $\begin{array}{c}5,54 b \\
6,7 b \\
36,7\end{array}$ & $\begin{array}{l}5,49 b \\
9,5 c \\
29,3\end{array}$ \\
\hline $\begin{array}{r}74,0 \\
498 a\end{array}$ & $\begin{array}{l}102,0 \\
552 b\end{array}$ & $\begin{array}{r}84,0 \\
600 c\end{array}$ & $\begin{array}{c}57,5 \\
568 \text { a b c }\end{array}$ \\
\hline $\begin{array}{l}10,79 a \\
16,4 a b \\
29,1 \\
43,1 a \\
785\end{array}$ & $\begin{array}{l}11,37 a \\
19,0 a \\
30,7 \\
47,6 b \\
81 !_{4}\end{array}$ & $\begin{array}{l}13,51 b \\
16,3 b \\
36,8 \\
47,1 b \\
846\end{array}$ & $\begin{array}{l}14,69 c \\
18,2 a b \\
31,2 \\
45,8 a b \\
780\end{array}$ \\
\hline 17,03 & 16,78 & 18,95 & 20,18 \\
\hline
\end{tabular}

Les chiffres suivis d'une lettre identique ne sont pas significativement différents $(\mathrm{P}<0,05)$.

\section{TABLEAU 7}

Essai 2: comparaison des 4 fourrages en quantité limitée

$y=$ temps d'ingestion ou de rumination en minutes

$x=$ quantité ingérée en $\mathrm{g} / \mathrm{kg} \mathrm{P}^{0,75}$

$n=6$

1. - Iiaisons entre durée d'ingestion et quantités ingérées

\begin{tabular}{l|l|l}
\hline Herbe $\ldots \ldots \ldots \ldots \ldots$ & $y=-19,7+4,6 x$ & $r=0,91^{*}$ \\
Foin $\ldots \ldots \ldots \ldots \ldots \ldots$ & $y=-8,8+4,9 x$ & $r=0,96^{* *}$ \\
Ensilage + acide $\ldots \ldots$ & $y=-20,6+5,2 x$ & $r=0,98^{* *}$ \\
Ensilage direct $\ldots \ldots \ldots$ & $y=-8,0+4,7 x$ & $r=0,98^{* *}$
\end{tabular}

2. - Liaisons entre durée de rumination et quantités ingérées

\begin{tabular}{|c|c|c|}
\hline Herbe & $y=131$ & $\mu=0,81^{*}$ \\
\hline Foin $\ldots \ldots \ldots$ & $y=88$ & $r=0,94 * *$ \\
\hline Ensilage + acide $\ldots \ldots$ & $y=83,5+17,0 x$ & $r=0,95^{* *}$ \\
\hline Ensilage direct $\ldots \ldots \ldots$ & $y=85,7+16,6 x$ & $r=0,91^{*}$ \\
\hline
\end{tabular}


confondues pour les trois fourrages conservés. Par contre, les paramètres de la droite de régression pour le fourrage vert sont significativement différents. Les durées unitaires d'ingestion sont en moyenne de 5,94 minutes par $\mathrm{g} / \mathrm{kg} \mathrm{P^{0,75 }}$ pour $1^{\prime}$ herbe, contre 4,32, 3,9I et 4,25 pour le foin et les deux ensilages.

Les durées de rumination pendant les 24 heures suivant la distribution du repas unique sont également reliées très étroitement aux quantités ingérées (tabl. 7) ; les droites de régression liant la durée de rumination et la quantité ingérée ne sont pas significativement différentes suivant les fourrages. Les durées unitaires de rumination sont pratiquement les mêmes pour les trois fourrages humides : 22,8 minutes par $\mathrm{g} / \mathrm{kg} \mathrm{P}^{0,75}$ pour l'herbe, 22,3 et 22,4 pour les deux ensilages contre 20,8 pour le foin.

\section{DISCUSSION}

Il ressort des deux essais que les moutons recevant de l'ensilage effectuent des repas plus courts que lorsqu'ils reçoivent le fourrage vert ou le foin correspondant. Cette différence se remarque en particulier plus nettement pour les repas suivant la distribution du fourrage aux animaux. En revanche, le nombre de repas augmente mais cela ne compense pas la diminution de leur durée. Il en résulte que la durée journalière d'ingestion des ensilages est plus courte que celle du fourrage vert ou du foin.

Par contre, la durée unitaire d'ingestion des ensilages, qui mesure l'inverse de la vitesse d'ingestion, varie de façon moins systématique : elle est, soit plus élevée, soit égale à celle du foin. L'élément majeur intervenant dans la diminution de la quantité ingérée par rapport au fourrage vert semble donc être la diminution de la durée journalière d'ingestion. Toutefois, cette diminution de la durée d'ingestion ne rend pas compte en totalité de la diminution de la quantité ingérée puisque parallèlement les animaux ingèrent plus ou moins rapidement. Notons que les différences de comportement observées s'établissent rapidement, en 4 à 5 jours pour les ensilages et I à 3 jours pour le foin. Elles doivent s'expliquer par des actions différentes des aliments, soit au niveau buccal, soit au niveau digestif, ou encore au niveau métabolique.

Ia faible longueur des repas d'ensilages est un fait intéressant et doit expliquer en partie l'augmentation de leur nombre.

Cette faible longueur est en revanche plus difficile à expliquer car elle peut avoir des causes diverses : qualités organoleptiques ou préhensibilité insuffisantes des ensilages, encombrement plus rapide du rumen avec les ensilages ou bien action de certains métabolites présents dans l'ensilage ou provenant de la digestion dans le rumen qui auraient un effet direct sur l'appétit. Pour faire la part de ces différentes causes il sera nécessaire de connaître plus exactement la quantité ingérée à chaque repas, en particulier au cours de ceux qui suivent la distribution de l'aliment, et le volume des contenus de rumen, avant et après ces repas.

La durée unitaire d'ingestion résulte à la fois de la réaction des animaux aux caractéristiques de 1'aliment (goût, odeur, préhensibilité, etc.) et de l'intensité de l'appétit. L'interprétation de ses variations est donc difficile. De plus, la durée uni- 
taire d'ingestion peut varier au cours du repas, d'un repas à l'autre, ou d'une période à l'autre : c'est le cas par exemple pour le foin entre les périodes I, 3 et 4 du premier essai, périodes entre lesquelles le nombre de repas a varié. Notons simplement que dans notre premier essai, lors des premiers jours de la distribution de l'ensilage, le goût et l'odeur du fourrage doivent expliquer la valeur relativement élevée de la durée unitaire d'ingestion, mais ne semblent pas être des facteurs limitant directement les quantités d'ensilages ingétées; en effet, parallèlement à la hausse de la durée unitaire d'ingestion, on note une forte augmentation du temps passé à ingérer de la part des animaux.

A la différence de l'ingestion, la rumination est peu modifiée par le mode de conservation du fourrage. Sa durée n'a été abaissée nettement que lorsque les animaux ont reçu le quart de leur ration normale. Tout se passe comme si la constance de la durée de rumination traduisait un certain besoin de ruminer de la part des animaux ; il faut que la quantité de fourrage dans le rumen soit très faible pour que cette constance soit affectée. La durée des cycles de rumination est plus élevée avec le foin qu'avec l'ensilage. Les variations de cette durée sont peut-être liées aux modifications de la structure physique du contenu, au degré de remplissage du rumen ou à la dureté du fourrage. Quoi qu'il en soit, la rumination ne semble pas être un facteur limitant la quantité ingérée puisque la durée unitaire de rumination peut varier dans des proportions très importantes pour le même fourrage lorsque le niveau d'ingestion varie. De plus, elle est la même pour les fourrages conservés différemment quand ils sont distribués en quantités faibles et égales.

Dans notre essai la vitesse de transit de l'indigestible ne semble pas avoir limité les quantités d'ensilage ingérées, mais il nous faudra mesurer la vitesse de disparition dans le rumen de la partie digestible des ensilages. Enfin, il est curieux de constater que la diminution de la quantité ingérée d'ensilage est progressive durant les jours qui suivent l'arrêt de la distribution du foin et s'amplifie au fur et à mesure que les animaux s'habituent à l'ensilage et l'ingèrent plus rapidement, mais cesse dès que les animaux reçoivent à nouveau du foin. Un encombrement progressif du rumen, ou l'action de certains métabolites, peuvent être à l'origine de ce phénomène.

L'étude des manifestations du comportement alimentaire et mérycique des moutons nous semble donc une voie intéressante pour aborder l'étude de la régulation de l'ingestion des animaux recevant des fourrages conservés. Il est évident que des mesures complémentaires doivent être effectuées en même temps pour évaluer plus précisément les effets des fourrages au niveau digestif et métabolique.

Reçu pour publication en mars 1972 .

\section{SUMMARY}

\section{STUDY OF SOME RELATIONSHIPS BETWEEN FORAGE CONSERVATION METHODS} AND FEEDING BEHAVIOUR AND RUMINATION IN SHEEP

I. The effect of the forage conservation method on the feeding behaviour and rumination of sheep was studied in the course of two trials.

- In the first trial, 5 sheep received hay and tall fescue silage of similar digestibilities during 6 successive periods according to threc methods : abrupt changes of diets based either on hay 
or silage both offered ad libitum; hay and silages in limited amounts at the same level of intake; hay and silage the same day either separately or at the same time.

- In the second trial, 6 sheep received the same timothy herbage either as green forage or as stored forage (one hay and two silages). Comparisons were made between ad libitum and restricted levels of feeding.

2. The daily time spent eating was shorter for silage than for green forage or for hay prepared from the same original herbage (tables 2 and 6). The lenght of silage meals was shorter and their number higher than in the case of hay or green forage.

3. The eating rate (opposite of the unitary eating time) of silage was variable : silage was eaten less rapidly than hay in the first trial and at the same rate in the second trial.

4. When the animals received silage without transition after a hay diet, the dry matter intake progressively decreased for 4 days whereas the unitary eating time and the number of meals abruptly increased (fig. I). When the animals were fed hay again, the dry matter intakc immediately increased as well as the length of the meal.

5. When the forages were offered ad libitum, the rumination time was relatively constant and did not seem to be a factor limiting the amount of silage eaten. Low and equal anounts of differently stored forages were ruminated in the same way. The lengths of the rumination cycles were shorter with silage or forage than with hay.

\section{RÉFÉRENCES BIBLIOGRAPHIQUES}

BALCH C. C., I950. Factors effecting the utilization of food by dairy cows : I. The rate of passage of food through the digestive tract. Brit. J. Nut. 4, 36r.

Demarquilly C., Jarrige R., i97o. The effect of method of forage conservation on digestibility and voluntary intake. Proc. 11th Inter. Grassl. Congr. 733-737. Surfers Paradise, Queensland, Australia.

Demarquilly C., Weiss Ph., I970. Tableaux de la valeur alimentaire des fourrages. S. E.I., étude $\mathrm{n}^{\circ} 42$.

Dulphy J. P., rg7r. Influence du poids vif et du niveau d'ingestion sur le comportement alimentaire et mérycique du mouton. Ann. Zootech. 20, 477-486.

Gordon J. G., I965. The relationship between rumination and the amount of roughages eaten by sheep. J. Agr. Sci. 64, I5I-r 55 .

Harris C. E., Raymond W. F., Wilson R. F., ig66. The voluntary intake of silage. Xth Inter. Grassl. Congress. Helsinki, p. 564 .

KerbaA A., I969. Etude de quelques facteurs de variation du comportement alimentaire et mérycique des ruminants. Thèse Doct. Sci. Clermont-Ferrand.

Mccarrick R. B., Maguire M. F., Poole D. B. R., Splllane I. A., I965. The nutritive value of ammonium bisulfate and molassed silages (III). Tr. J. Agr. Res. 4, I 35 .

McLeod D. S., Wilkins R. J., Raymond W. F., I970. The voluntary intake by sheep and cattle of silages differing in free-acid content. J. Agr. Sci. \%5, 3II-3I9.

MCLEOD D. S., WiLkins R. J., I970. The effect of intra animal feeding on the intake of silage. $J$. Agric. Sci. Camb. 75, 559 .

Moore L. A., Thomas J. W., Svkes J. F., 1960. The acceptability of grass legume silage by dairy cattle. VIIIth Int. Grassl. Congress. Reading p. 7or.

RUCKEBUSCH, I963. Recherches sur la régulation centrale du comportement alimentaire des numinants. Thèse Doct. Sci. Lyon.

Thomas J. W., Moore L. A., Okamoto M., Sykes J. F., I960. A study of factors affecting rate of intake of heifers fed silage. J. Dairy Sci. 44, I471.

Waldo D. R., Milier R. W., Smith L. D., Okamoto M., Moore L. A., ig66. The effect of direct cut silage compared to hay, on intake digestibility, $\mathrm{N}$ Utilisation, heifer growth and rumen retention. Xth Inter. Grassl. Congress. Helsinki, p. 750.

WeLch J. G., Sмiтh A. M., Ig69a. Influence of forage quality on rumination time in sheep. $J$. Anim, Sci, 28, 81 3-818.

Welch J. G., Sмıтн A. M., I969 $b$. Effect of varying amounts of forage intake on rumination. $J$. Anim. Sci. 28, 827-830. 